8:39 AM 11/22/2002

LBNL-51736

\title{
Effects of the Amorphous Oxide Intergranular Layer Structure and Bonding on the Fracture Toughness of a High Purity Silicon Nitride
}

\author{
A. Ziegler ${ }^{l}$, C. Kisielowski ${ }^{2}$, M. J. Hoffmann ${ }^{3}$, and R. O. Ritchie ${ }^{l}$ \\ ${ }^{1}$ Lawrence Livermore National Laboratory, University of California, Livermore, CA 94551 \\ ${ }^{2}$ Lawrence Berkeley National Laboratory, University of California, Berkeley, CA 94720 \\ ${ }^{3}$ IKM, University of Karlsruhe, D-76131 Karlsruhe, Germany
}

November 2002

submitted to

MRS Symposium on Structure-Property Relationships of Oxide Surfaces and Interfaces

Fall MRS Meeting, December 2002

Work supported by the Director, Office of Science, Office of Basic Energy Sciences, Division of Materials Sciences and Engineering of the U.S. Department of Energy under Contract No. DEAC03-76SF00098 


\title{
Effects of the Amorphous Oxide Intergranular Layer Structure and Bonding on the Fracture Toughness of a High Purity Silicon Nitride
}

\author{
A. Ziegler ${ }^{1}$, C. Kisielowski ${ }^{2}$, M. J. Hoffmann ${ }^{3}$ and R. O. Ritchie ${ }^{2}$ \\ ${ }^{1}$ Lawrence Livermore National Laboratory, University of California, Livermore, CA 94551, USA \\ ${ }^{2}$ Lawrence Berkeley National Laboratory, University of California, Berkeley, CA 94720, USA \\ ${ }^{3}$ IKM, University of Karlsruhe, D-76131 Karlsruhe, Germany
}

\begin{abstract}
The microstructural evolution and structural characteristics and transitions in the thin grainboundary oxide films in a silicon nitride ceramic, specifically between two adjacent grains and not the triple junctions, are investigated to find their effect on the macroscopic fracture properties. It is found that by heat treating a model $\mathrm{Si}_{3} \mathrm{~N}_{4}-2 \mathrm{wt} \% \mathrm{Y}_{2} \mathrm{O}_{3}$ ceramic for $\sim 200 \mathrm{hr}$ at $1400^{\circ} \mathrm{C}$ in air, the fracture toughness can be increased by $\sim 100 \%$, coincident with a change in fracture mechanism from transgranular to intergranular. Structural phase transformations occur in the thin grain boundaries during oxidation that are revealed by XRD, EDX, Raman and EELS analyses. They affect the local bonding of atoms. It is concluded that only specific crystal "building blocks", i.e., tetrahedra, are transformed along the grain boundary and the resulting difference in the atomic structure of the oxide interface is seen directly to alter the macroscopic fracture behavior.
\end{abstract}

\section{INTRODUCTION}

Minute structural changes and transitions along the thin grain-boundary films in silicon nitride ceramics can have a marked influence on macroscopic behavior. Indeed, post-sintering heat treatment of $\mathrm{Si}_{3} \mathrm{~N}_{4}$ ceramics has been used to improve their mechanical properties, specifically by fully or partially crystallizing the amorphous triple junction regions $[1,2]$, although the mechanism by which, for example, the fracture toughness is increased is uncertain. Triple junctions and thin grain boundaries are interconnected and thus their respective transformations and chemical balances can influence each other. However, it is not clear what happens in terms of atomic structure and bonding at the very local level of the thin grain boundaries. One can propose that the local bonding character and specific structural transitions along the thin two-grain interface are responsible for the marked changes in the mechanical response. During an oxidative heat treatment, it is known that oxygen diffuses into the bulk material while sintering additive cations, and possibly also nitrogen, diffuse outward to the surface. It is also probable that oxygen replaces nitrogen along the thin grain boundary, consistent with calculated binding energies of Si-clusters with various $\mathrm{O} / \mathrm{N}$ compositions, which show that $\mathrm{Si}-\mathrm{O}$ is the more favorable bond to $\mathrm{Si}-\mathrm{N}$ [3]; this implies that a low $\mathrm{N}: \mathrm{O}$ ratio strengthens the atomic bonding along the boundary. However, Becher et al. [4] has observed that a decrease in $\mathrm{N}: \mathrm{O}$ ratio apparently reduces the interfacial debonding energy for $\beta-\mathrm{Si}_{3} \mathrm{~N}_{4}$ whiskers embedded in a $\mathrm{Si}-\mathrm{Al}-\mathrm{Y}$ oxynitride glass. Furthermore, other sintering additives used to densify $\mathrm{Si}_{3} \mathrm{~N}_{4}$ have been found to affect the complex intergranular bonding state too.

In the present study $\mathrm{Y}_{2} \mathrm{O}_{3}$ was used to densify silicon nitride. High yttrium concentrations were expected in the thin glassy grain-boundary phase since $\mathrm{Y}$ is insoluble in $\beta-\mathrm{Si}_{3} \mathrm{~N}_{4}$. The specific role of the $\mathrm{Y}_{2} \mathrm{O}_{3}$ additions, and more importantly, the subsequent oxidative heat treatments on the mechanical properties are investigated. With the as-sintered material taken as a 
reference, the microstructural evolution of the thin grain-boundary phase during the $1400^{\circ} \mathrm{C}$ oxidative heat treatment is examined to discern some of the structural transitions and transformations in atomic bonding that cause the marked changes in toughness properties. Only the thin grain boundary regions are examined as this is the site of the most interesting phenomena.

\section{EXPERIMENTAL PROCEDURES}

The silicon nitride under study was fabricated via a two-step sintering technique, consisting of a dilatometer-controlled gas-pressure-sintering and a successive hot-isostatic pressing densification [5]. This highly pure and controlled processing route allows for an almost impurityfree densification, thus permitting an unambiguous investigation of the role of small quantity sintering elements. The microstructure consisted of acicular-shaped (mainly $\beta-\mathrm{Si}_{3} \mathrm{~N}_{4}$ ) grains (average length of $5 \mu \mathrm{m}$, aspect ratio of $8: 1)$ and equiaxed grains $(0.5-1.5 \mu \mathrm{m}$ in size). $25 \mathrm{~mm}$ diameter, $3 \mathrm{~mm}$ thick disc samples were heat treated in air at $1400^{\circ} \mathrm{C}$ for $25,50,100$ and $200 \mathrm{~h}$; the non-heat-treated samples served as a reference material.

Fracture toughness $K_{\mathrm{c}}$ values were estimated on polished samples using $10 \mathrm{~kg}$ Vickers indents. An average of ten indents on two bulk samples of each material was evaluated.

X-ray diffraction (XRD) was used (Siemens D5000, Diffrac AT, Siemens, Germany) on fracture surfaces to identify phases in the as-sintered ceramic and for monitoring phase changes after each heat treatment cycle. Raman spectroscopy was performed using an integrated Raman microscope system (Labram by ISA Groupe Horiba), with a $\sim 3 \mu \mathrm{m}$ diameter internal HeNe laser beam $(\lambda=632 \mathrm{~nm})$. Line scan spectra were recorded on a polished mid-plane of each sample using scans $30 \mu \mathrm{m}$ in length to cover both grains and grain boundaries. Electron energy-loss spectroscopy (EELS) was carried out on thin TEM samples in a Philips CM200/FEG TEM to discern the distribution of chemical elements along and across the boundaries; a PEELS (parallel EELS) spectrometer (Gatan Inc., Pleasanton, Calif.) was used with an energy resolution of $1.0 \mathrm{eV}$. The resulting spectra were subject to the 'spatial difference' method [6]. High-resolution transmission electron microscopy (HRTEM) was conducted using a Philips CM300/FEG/UT, equipped with a field-emission electron source (FEG) and an ultra-twin objective lens of low spherical and chromatic aberrations. This microscope was modified to improve its information limit, such that lattice images can be recorded aberration free to a smallest distance of about $0.8 \AA$ [7]. Scanning electron microscopy (SEM) of the fracture surfaces was performed using a JEOL 6300 scanning electron microscope.

\section{RESULTS AND DISCUSSION}

Results are presented in terms of the macroscopic mechanical behavior, which is then related to the structural characteristics and transitions in the grain-boundary phase. Structural analysis is performed with experiments of increasing spatial resolution starting with XRD and eventually using phase-reconstructed HRTEM imaging. All of the applied experiments reveal finger prints of the ongoing transformations.

In Fig. 1a, it can be seen that aging for more than $50 \mathrm{~h}$ at $1400^{\circ} \mathrm{C}$ in air leads to a $\sim 100 \%$ increase in the fracture toughness, coincident with a change in fracture mode from transgranular in the as-sintered $\mathrm{Si}_{3} \mathrm{~N}_{4}$ to intergranular after aging (Figs. 1b,c). The toughening results from debonding along the grain-boundary phase leading to an intergranular crack path, which in turn induces crack-tip shielding due to the bridging of the interlocking grains $[8,9]$. 


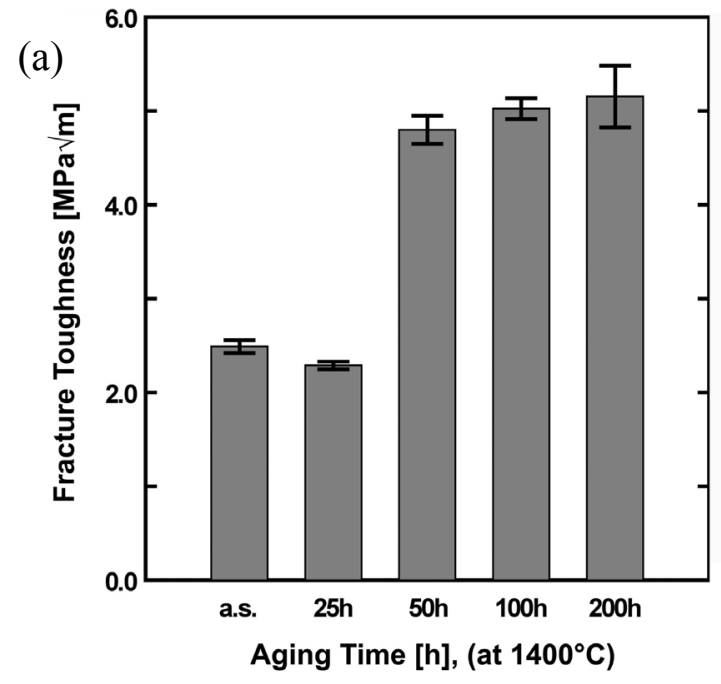

(b)

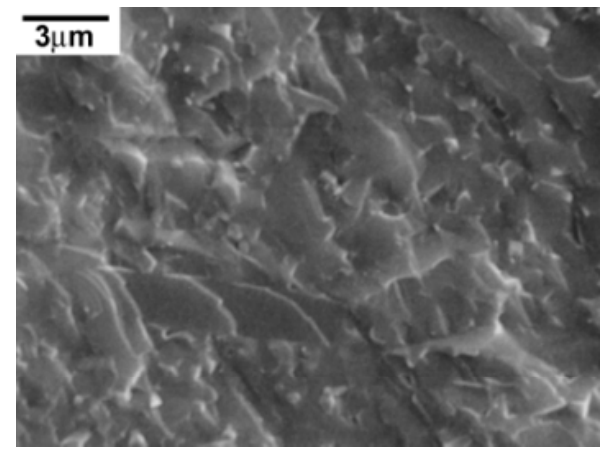

(c)

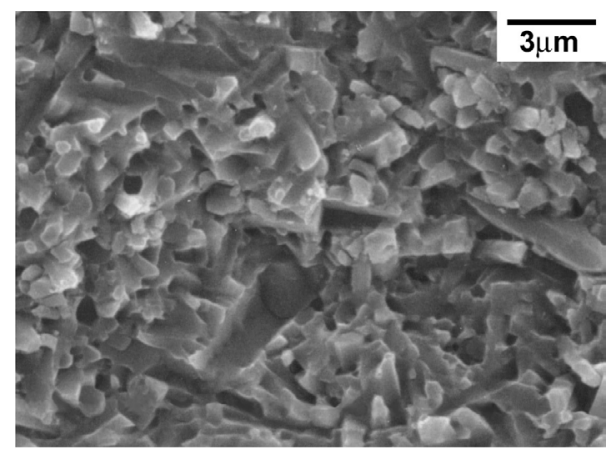

Figure 1 (a) Indentation fracture toughness as a function of aging time. (b) and (c) show SEM micrographs of fracture surfaces of the as-sintered and $200 \mathrm{~h}$ heat treated sample, respectively.

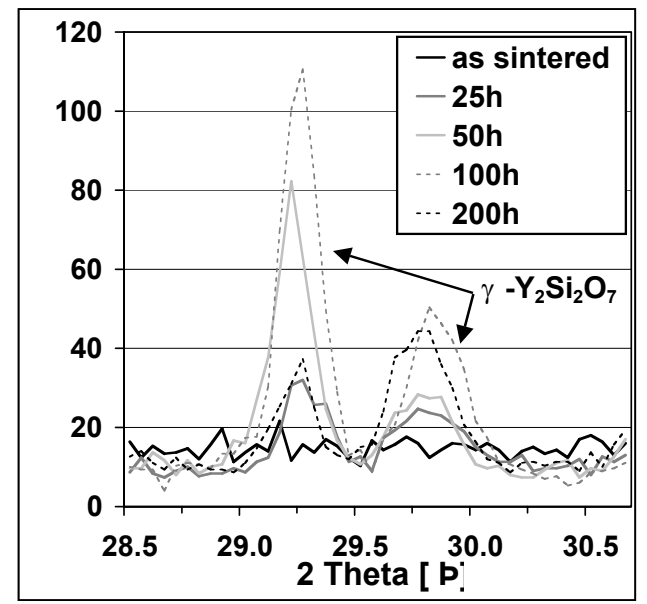

Figure 2 Region of interest of the XRD spectrum showing the changes in the $\gamma-\mathrm{Y}_{2} \mathrm{Si}_{2} \mathrm{O}_{7}$ peak throughout the heat treatment. Note that only a very small portion of the full XRD spectrum is shown.

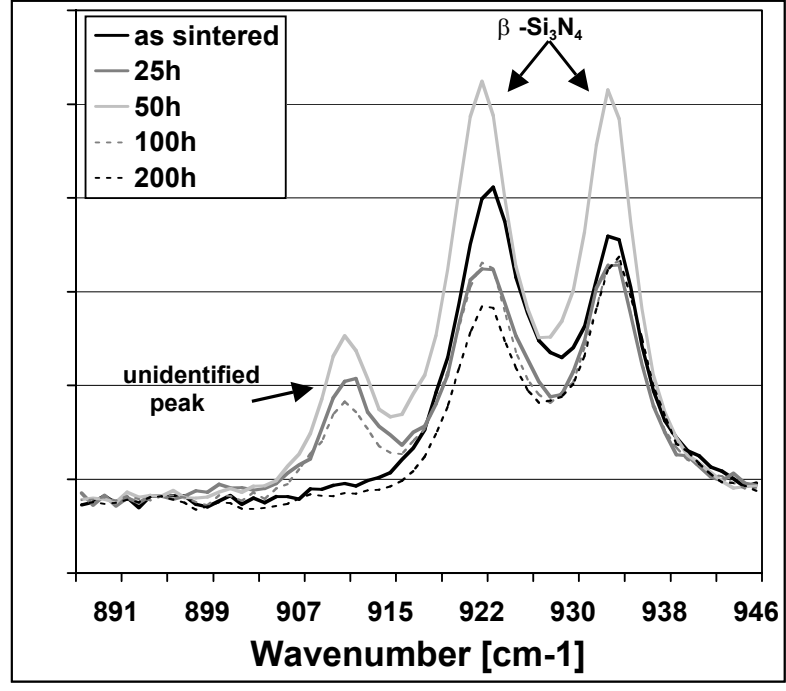

Figure 3 Region of interest in the Raman spectrum, demonstrating changes in atomic bonding during the heat treatment.

Debonding and intergranular fracture implies that the atomic bonds along the boundary are not as strong as those within the grains. The interface strength, however, is determined by the composition and degree of local order and bonding in the thin grain-boundary film. The observed change in fracture mechanism can be associated with structural transformations of this secondary phase, that incorporate $\mathrm{O}, \mathrm{N}$ and the sintering-aid cations, or with the relocation of these ions. As the present $\mathrm{Si}_{3} \mathrm{~N}_{4}$ was simply heat treated, it can be assumed that $\mathrm{O}$ is the major element that 
diffuses inward while $\mathrm{Y}$ diffuses outward. Since the diffusion distances along the grain boundaries for these elements at these temperatures are only a few hundred micrometers from the outer specimen surface, the possible variation in bonding at the specimen mid-plane would be limited. Consequently, we assume that relocation of elements is more important than in or out diffusion from the entire sample. Our focus is thus on oxygen and yttrium short-range relocation.

All peaks in XRD spectra of the as-sintered ceramic could be identified with $\beta-\mathrm{Si}_{3} \mathrm{~N}_{4}$. Figure 2 shows a very small portion of the full XRD spectrum which is of interest. An oxynitride phase $\mathrm{Si}_{2} \mathrm{~N}_{2} \mathrm{O}$ and a yttrium disilicate phase $\gamma-\mathrm{Y}_{2} \mathrm{Si}_{2} \mathrm{O}_{7}$ form with aging. Significant dissolution of $\gamma-$ $\mathrm{Y}_{2} \mathrm{Si}_{2} \mathrm{O}_{7}$ occurs after $\sim 100 \mathrm{~h}$, (see observed peak at $2 \theta=29.22^{\circ}$ ) for reasons that remain unclear.

Results of a Raman spectroscopy analysis are presented in Fig. 3 (only a small portion of the full spectrum is shown). All of the peaks in the spectra could be identified with pure $\beta-\mathrm{Si}_{3} \mathrm{~N}_{4}$, except one peak located at $910.67 \mathrm{~cm}^{-1}$, which could not be identified with the vibrational modes of any particular Y-disilicate polymorph nor $\mathrm{Si}_{2} \mathrm{~N}_{2} \mathrm{O}$. Akin to the $\gamma-\mathrm{Y}_{2} \mathrm{Si}_{2} \mathrm{O}_{7}$ peak in the XRD spectrum, this peak appears and disappears during the course of the heat treatment; it can be detected after 25,50 and $100 \mathrm{~h}$ at $1400^{\circ} \mathrm{C}$ but completely disappears after $200 \mathrm{~h}$. This suggests a relation of the $910.67 \mathrm{~cm}^{-1}$ mode with the presence of $\gamma-\mathrm{Y}_{2} \mathrm{Si}_{2} \mathrm{O}_{7}$, even though a precise identification could not be achieved.

Results of the EELS analysis are shown in Fig. 4, with the Si- $L_{2,3}$ edge being of special interest. The top two curves represent the spectra from a thin $\mathrm{Si}_{3} \mathrm{~N}_{4}$ grain boundary and a matrix grain; the lower two curves are $\mathrm{Si}-L_{2,3}$ spectra of $\mathrm{Si}_{2} \mathrm{~N}_{2} \mathrm{O}$ and $\mathrm{SiO}_{2}$. The curves at the bottom highlight differences induced by each heat treatment cycle (reference is the as-sintered material). Spectra from the boundary and the grains show only small but important differences that are well reproducible in successive pairs of measurements. In contrast, the difference of the $\mathrm{Si}_{2} \mathrm{~N}_{2} \mathrm{O}$ and the $\mathrm{SiO}_{2}$ spectra is clear, as the $\mathrm{Si}$ edge is shifted a few eV and additional features appear in the two spectra.

Averaging all recorded spectra reduces noise, and employing the spatial difference method permits an assessment of differences in chemical bonding and atomic structure between the grain boundary and the matrix. The lowest curves in Fig. 4 correspond to the individual stages of heat treatment. Although the as-sintered material exhibits only slight differences between the grain and the boundary, peaks appear and disappear upon heat treatment akin to the X-ray and Raman analyses. The peak located at $\sim 107 \mathrm{eV}$ does not show up until after annealing for $100 \mathrm{~h}$ at $1400^{\circ} \mathrm{C}$. Another peak at $\sim 118 \mathrm{eV}$ appears immediately after $25 \mathrm{~h}$ aging but disappears at longer aging times until after $200 \mathrm{~h}$, when it reaches again the level of the as-sintered sample.

The spectra of $\mathrm{Si}_{2} \mathrm{~N}_{2} \mathrm{O}$ and $\mathrm{SiO}_{2}$ serve to identify some of these changes. Specifically, the peak in the difference spectrum at $\sim 107 \mathrm{eV}$ can be associated with $\mathrm{Si}_{2} \mathrm{~N}_{2} \mathrm{O}$ in the boundary. The other peak at $\sim 118 \mathrm{eV}$ does not fully correspond to, nor align with, the $\mathrm{Si}_{2} \mathrm{~N}_{2} \mathrm{O}$ or $\mathrm{SiO}_{2}$ spectra. At this point it can only be speculated that $\mathrm{Y}$ or a combination of $\mathrm{SiO}_{2}$ and $\mathrm{Y}_{2} \mathrm{O}_{3}$ is involved since only $\mathrm{Si}, \mathrm{O}, \mathrm{N}$ and $\mathrm{Y}$ are in the boundaries. However, it is clear that a change in atomic bonding occurs.

Having established that a structural transformation occurs during an oxidative heat treatment, the next question is how the observed phases are connected to the matrix grain. Calculations $[10,11]$ to evaluate the atomic position of yttrium in $\mathrm{Si}_{3} \mathrm{~N}_{4}$ grain boundaries suggest that $\mathrm{Y}$ and other larger rare-earth elements show a tendency to attach to the nitrogen-terminated prismatic plane of the $\beta-\mathrm{Si}_{3} \mathrm{~N}_{4}$ grains. These calculations presume that incomplete Si-hexagons reach into the amorphous grain boundary, consistent with the current HRTEM results on the boundaries which confirm that Si-half-rings do indeed protrude into the amorphous grain boundary (Fig. 5) 
for potential $\mathrm{Y}$ ion attachment. However, the presence of $\mathrm{Y}$ ions at such sites remains to be investigated further [12]. From these analyses, we conclude that $Y$ can attach to the prism plane sites and can extend via a modified yttrium disilicate type of bonding further into the thin amorphous grain boundary.

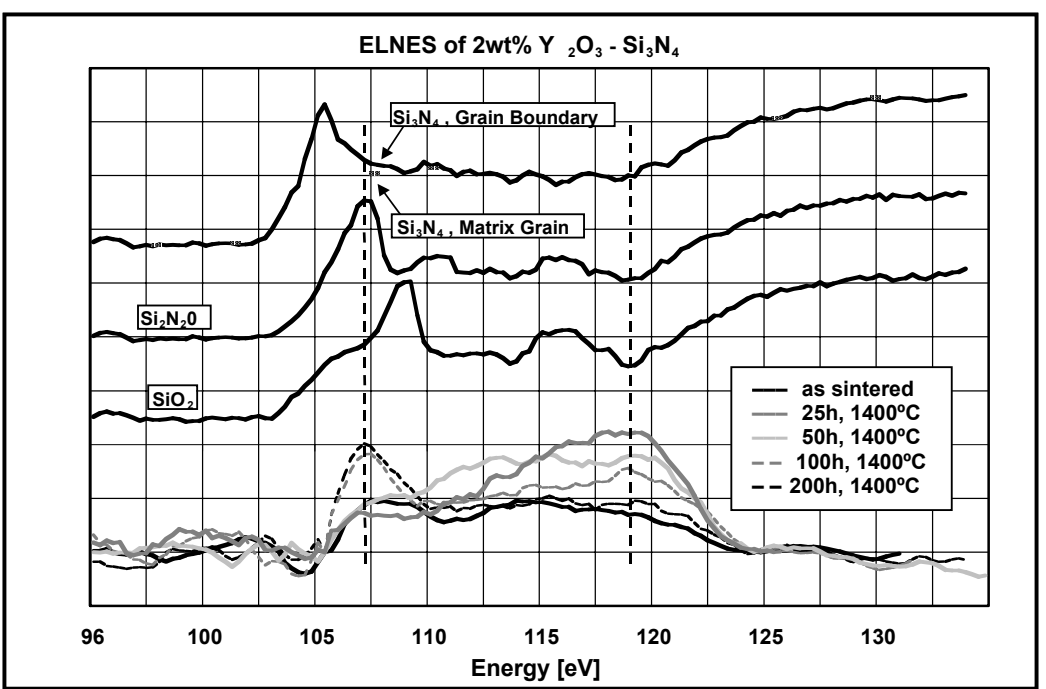

Figure 4 EELS spectrum. Averaging over twenty EELS spectra on and off the grain boundary and employing then the 'spatial difference' method, where the matrix grain spectrum is weighted and subtracted from the grain boundary spectrum, one obtains the true grain boundary EELS spectrum. For visual simplicity, the weighing factor here was taken as 1.00 instead of $\sim 0.85$, as seen sometimes in other investigations. (Intensity axis is not to scale).

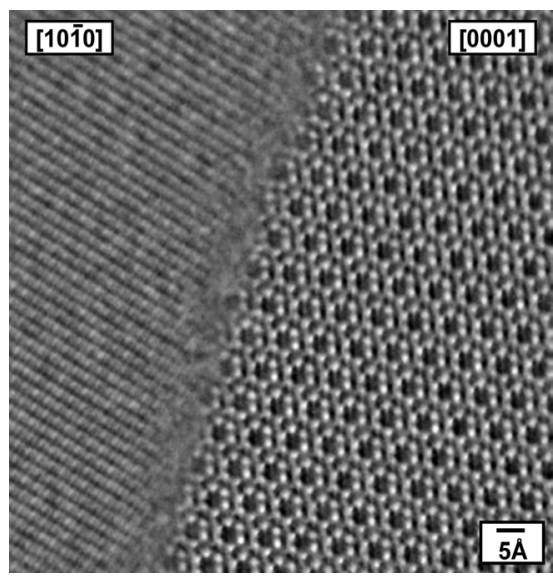

Figure 5 HRTEM micrograph of the $200 \mathrm{~h}$ heat-treated sample, showing the grain boundary between two $\mathrm{Si}_{3} \mathrm{~N}_{4}$ matrix grains with their respective orientations. The grain boundary is amorphous and approximately 5 to $7 \AA$ thick.

Based on crystallographic data of known yttrium disilicate polymorphs [13], individual $\mathrm{Y}_{2} \mathrm{Si}_{2} \mathrm{O}_{7}$ crystals are unlikely to form, because they would almost completely bridge the width of the thin boundary. Moreover, they would need to conform to bonding characteristics on the other side of the boundary to the adjacent $\mathrm{Si}_{3} \mathrm{~N}_{4}$ matrix grain, which usually has no orientation relationship with the first grain. The average grain boundary thickness measured in the present samples ranged from 5 to $10 \AA$, while the unit cell dimensions for $\gamma-\mathrm{Y}_{2} \mathrm{Si}_{2} \mathrm{O}_{7}$ are (in $\AA$ ): $a=5.579$, $b=10.857, c=4.696$ and for $\mathrm{Si}_{2} \mathrm{~N}_{2} \mathrm{O}, a=8.843, b=5.473$, and $c=4.835$ [14]. However, the $\mathrm{Si}_{2} \mathrm{~N}_{2} \mathrm{O}$ features in the EELS difference spectra indicate that a $\mathrm{Si}_{2} \mathrm{~N}_{2} \mathrm{O}$-specific type of atomic bonding state is present along the grain boundary. Since full individual crystal unit cells cannot form, simply due to geometric constraints, we thus tentatively conclude that only characteristic "building blocks or units", i.e., tetrahedra, of the identified crystal structures form along the thin grain boundaries. This could allow sintering aide ions and oxidation products to be incorporated into the amorphous grain-boundary film.

\section{CONCLUSIONS}

Changes in thin grain boundary chemistry and the very local atomic bonding are key factors to understanding the observed $100 \%$ increase in toughness of a silicon nitride containing $2 \mathrm{wt} \%$ 
$\mathrm{Y}_{2} \mathrm{O}_{3}$ (concomitant with a change from transgranular to intergranular fracture) after aging for 200 $\mathrm{h}$ in air at $1400^{\circ} \mathrm{C}$. Using XRD, Raman spectroscopy and EELS, a range of structural transformations was found to occur during the oxidative heat treatment that are associated with the $\mathrm{Si}_{2} \mathrm{~N}_{2} \mathrm{O}$ and $\gamma-\mathrm{Y}_{2} \mathrm{Si}_{2} \mathrm{O}_{7}$ phases. Specifically, the formation and dissolution of these phases within the thin grain-boundary regions was revealed. HRTEM imaging in combination with recent theoretical calculations revealed the possibility for yttrium ion attachment at the prism planes. However, owing to geometric constraints, full individual crystals of the detected phases are not able to form. We conclude that only specific "building blocks", i.e., tetrahedra, can form and transform along the grain boundary, and that these in turn must exhibit a specific type of bonding for the detected phases.

\section{ACKNOWLEDGEMENTS}

This work was supported by the Director, Office of Science, Office of Basic Energy Sciences, Division of Materials Sciences and Engineering of the U.S. Department of Energy under contract No. DE-AC03-76SF00098. The authors thank E. C. Nelson (NCEM) for help with the TEM studies.

\section{REFERENCES}

1. F. F. Lange, B. I. Davis, M. G. Metcalf, J. Mat. Sci. 18, 1497 (1983).

2. M. K. Cinibulk, G. Thomas, S. M. Johnson, J. Am. Ceram. Soc. 73, 1606 (1990).

3. E. Y. Sun, P. F. Becher, C. H. Hsueh, G. S. Painter, S. B. Waters, S. L. Hwang. M. J. Hoffmann, Acta Mat. 47, 2777 (1999).

4. P. F. Becher, E. Y. Sun, C. H. Hsueh, K. B. Alexander, S. L. Hwang, S. B. Waters, C. G. Westmoreland, Acta Mat. 44, 3881 (1996).

5. M. J. Hoffmann, A. Geyer, R. Oberacker, J. Euro. Ceram. Soc. 19, 2359 (1999).

6. H. Müllejans, J. Bruley, Ultramicroscopy 53, 351 (1994).

7. Y. C. Wang, A. Fitzgerald, E. C. Nelson, C. Song, M. A. O’Keefe, C. Kisielowski, Microscopy and Microanalysis 5, 822 (1999).

8. S. M. Wiederhorn, Annual Review of Materials Science 14, 373 (1984).

9. R. O. Ritchie, Mater. Sci. Eng. A103, 15 (1988).

10. T. Nakayasu, T. Yamada, I. Tanaka, H. Adachi, S. Goto, J. Am. Ceram. Soc. 81, 565 (1998).

11. G. S. Painter, P. F. Becher, W. A. Shelton, private communication (2002).

12. A.Ziegler, C.Kisielowski, M.J.Hoffmann, R.O.Ritchie, J. Am. Ceram. Soc. (2002) in review.

13. J. Felsche, Naturwissenschaften 57, 127 (1970).

14. I. Idrestedt, C. Brosset, Acta Chem. Scan. 18, 1879 (1964). 